\title{
The utility of transrectal sonoelastography in preoperative prostate cancer assessment
}

\author{
Steffen Rausch ${ }^{1}$, Wibke Alt ${ }^{2}$, Hartmut Arps ${ }^{2}$, Berthold Alt ${ }^{1}$, Tilman Kälble ${ }^{1}$ \\ ${ }^{1}$ Department of Urology, ${ }^{2}$ Department of Pathology, Klinikum Fulda, Fulda, Germany
}

\begin{abstract}
Aim: To determine the diagnostic quality of transrectal sonoelastography (SE) in the prediction and localisation of prostate cancer, we prospectively examined patients treated with radical prostatectomy in our urology department. Methods: From April 2010 to January 2011, 61 patients with biopsy-proven prostate cancer underwent preoperative transrectal gray-scale (bmode) ultrasound and SE of the prostate. Cancer-suspicious areas were documented for b-mode and SE, dividing the prostate into six topographic sectors. Suspicious areas in both modalities were compared to tumour localisation in the prostatectomy specimen. Sensitivity, specificity, positive- and negative predictive values were calculated for both investigation techniques. Results: Prostate cancer was present in 232 of 366 pathological sectors (62\%). B-mode ultrasound showed 113 suspicious sectors, while SE indicated prostate cancer in 157 areas. The precise localisation of at least one pathologically confirmed cancerous lesion was possible in $42 / 61$ (69\%) patients by b-mode ultrasound and 56/61 (92\%) patients by $\mathrm{SE}(\mathrm{P}<0.005)$. The sensitivity for b-mode ultrasound was $33 \%$ and specificity $74 \%$. For SE sensitivity was $53 \%$, while specificity was 74 $\%$. Conclusions: SE offers a more precise localisation of prostate carcinoma than conventional ultrasound. To investigate the possible advantages of SE in during prostate biopsy and its value in the prediction of extracapsular cancer further studies are required.
\end{abstract}

Keywords: prostate cancer, elastography, ultrasound, prostatectomy

\section{Introduction}

As continuous efforts are undertaken to improve prostate cancer diagnosis, imaging represents a diagnostic sector with a high potential to offer relevant information on cancer staging and therapy planning. Within the setting of prostate biopsy, improved imaging modalities could assist investigators in identifying and localising cancer suspicious areas for targeted samples. So far, no imaging modality has established itself as practical in a broad clinical routine to close these diagnostic gaps, and

Received 11.04.2012 Accepted 29.05.2012

Med Ultrason

2012, Vol. 14, No 3, 182-186

Corresponding author: Steffen Rausch MD,

Department of Urology, Klinikum Fulda gAG, Fulda, Germany

Pacelliallee 4, D-36043 Fulda,

Phone: $+49(0) 661-845951$

Fax: $+49(0) 661-845952$

Email: Steffen.Rausch@klinikum-fulda.de meanwhile clinical studies are being conducted to evaluate various diagnostic procedures. To date, 10-12 core randomised prostate biopsy remains the gold standard for cancer diagnosis [1-3], with acceptance of its known limitations.

Sonoelastography (SE) has recently been described as an emerging imaging modality for prostate carcinoma [4-9]. As available data on SE are merely based on Hitachi technology and heterogeneous towards patient numbers and sensitivities, we prospectively evaluated our experience using an ultrasound device from an alternative manufacturer (Siemens).

\section{Methods}

From April 2010 to January 2011, 61 patients with biopsy-proven prostate carcinoma designated to undergo radical prostatectomy at our Department of Urology were preoperatively examined by transrectal gray-scale (b-mode) ultrasound and transrectal SE. A Siemens 
Acuson S2000 ultrasound device with an EC9-4 transrectal transducer and eSie Touch ${ }^{\mathrm{TM}}$ elasticity imaging was used to conduct the studies. Written informed consent was obtained by all participating patients. All patients were investigated by the same examiner who was blinded to all preoperatively available data, such as level of prostate specific antigen (PSA), digital rectal examination, and the pathological reports of prior prostate biopsies. Patients were examined in the left lateral position. In order to validate the potential of localising prostate carcinoma in both imaging modalities, suspicious areas were documented in six topographic sectors, left and right prostate apex, mid gland and base. Areas were classified as suspicious, when a demarked, hypoechogenic pattern was present in gray-scale imaging. Elastographic images were generated by soft and rhythmic compression of the prostate using the ultrasound transducer.

Tumour suspicion was defined as a demarked section of increased tissue stiffness, suggested by colour scaling and a particular quality factor of the elastography software (>70), indicating quality of compression and reproducibility of images.

In both imaging modalities, the investigation focused on the peripheral zone of the prostate. Furthermore, prostate size and morphological suspect of capsular excess or seminal vesicle invasion were recorded. The included patients all underwent open radical retro-pubic prostatectomy with bilateral iliac lymph node dissection. The histological analysis was performed by two pathologists. Prostatectomy specimens were step sectioned and processed according to a standard protocol. Postoperatively the localisation of suspicious areas in prostate imaging was compared to the pathology results. Sensitivity, specificity, positive- (PPV) and negative predictive values (NPV) for conventional ultrasound and SE were calculated. To evaluate the influence of prostate size on diagnostic quality, we analysed sensitivity in dependence of prostate volume. A chi-square test was used to determine statistical significance.

\section{Results}

We prospectively examined 61 patients with prostate carcinoma prior to open radical retro-pubic prostatectomy. Median preoperative PSA was $6.40 \mathrm{ng} / \mathrm{ml}$. The mean age at surgery was 63.8 years. Median prostate size measured $37 \mathrm{~cm}^{3}$, with a range of $16 \mathrm{~cm}^{3}$ to $78 \mathrm{~cm}^{3}$. In conventional gray-scale ultrasound a total of $113 \mathrm{sec}-$ tors were classified as suspicious for prostate cancer, SE indicated prostate cancer in 157 areas. Pathological analysis detected prostate cancer in 232 of 366 sectors
$(62 \%)$. Histological tumour detection was most prominent in the mid gland area (44\%), apical carcinoma was detected in $38 \%$, while basal tumours occurred in $18 \%$ of all cases. There was no difference regarding the side of tumour incidence. Figure 1 shows an exemplary examination picture and the corresponding postoperative histological analysis. Patient characteristics and postoperative stage distribution are shown in table I. The correct detection of at least one pathologically confirmed tumour lesion (index tumour) was possible for 56 patients (92\%) by SE, whereas conventional ultrasound predicted cancer localisation correctly in 42 $(69 \%)$ patients $(P<0.005)$. Overall, the sensitivity for the correct localisation of prostate cancer was $33 \%$ using gray-scale ultrasound. The specificity accounted for $74 \%$, the respective positive predictive value and negative predictive values were $70 \%$ and $38 \%$. SE allowed

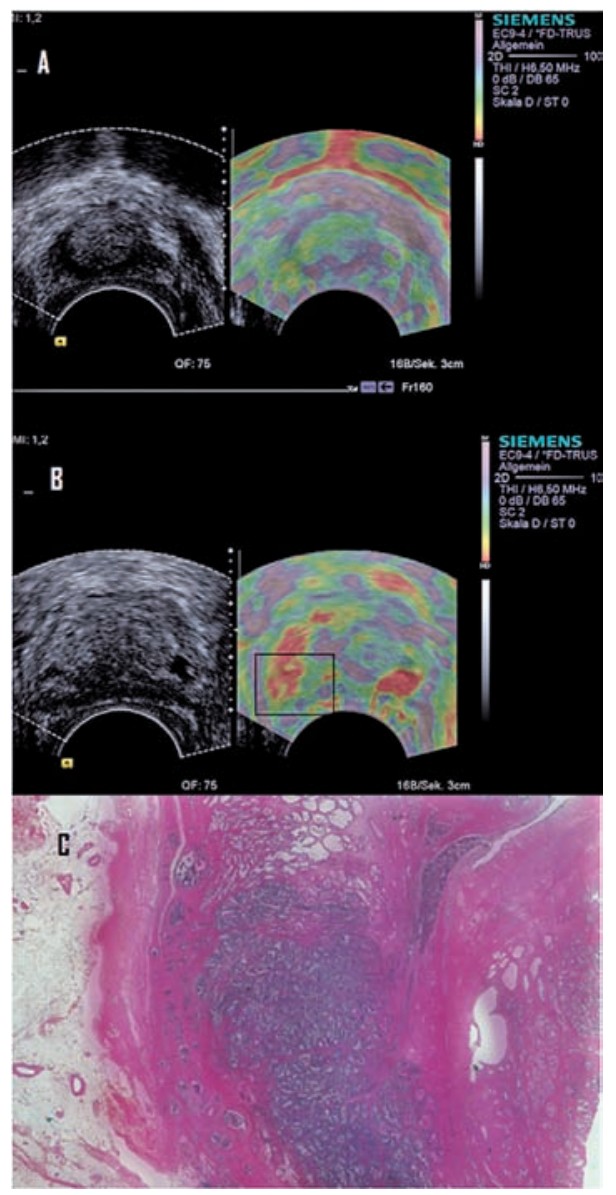

Fig 1. Examination pictures and histopathological analysis - A: elastographic image without pathological findings at the prostate apex/urethral intersection (QF:75=quality factor) - B: suspicious area in the right mid-gland section, the marked sector (box) is shown in C (QF:75)- C: histological slide with confirmation of prostate cancer according to $\mathrm{B}$. 
Table I. Patient characteristics.

\begin{tabular}{lcc}
\hline Age at surgery, mean (IQR) & & $63.8 \mathrm{y}(59.02-69.81 \mathrm{y})$ \\
PSA. median (IQR) & & $6.4 \mathrm{ng} / \mathrm{ml}(4.97-8.75 \mathrm{ng} / \mathrm{ml})$ \\
Prostate volume, median (range) & & $37 \mathrm{cc}(16-79 \mathrm{cc})$ \\
Clinical staging & $\mathrm{T} 1$ & $39(64 \%)$ \\
& $\mathrm{T} 2$ & $20(33 \%)$ \\
& $\mathrm{T} 3$ & $2(3 \%)$ \\
Pathological staging & pT2 & $40(66 \%)$ \\
& pT3a & $15(25 \%)$ \\
& pT3b & $6(10 \%)$ \\
Pathological Gleason Score & pT4 & - \\
& $\leq 6$ & $37(61 \%)$ \\
& $7 \mathrm{a}$ & $8(13 \%)$ \\
& $7 \mathrm{~b}$ & $8(13 \%)$ \\
Nodal status & $\geq 8$ & $8(13 \%)$ \\
& $\mathrm{N} 0$ & $57(93 \%)$ \\
& $\mathrm{N}+$ & $4(7 \%)$ \\
\hline
\end{tabular}

$\mathrm{IQR}=$ inter-quartile range

Table II. Sensitivity, specificity, positive and negative predictive values for correct localisation of prostate cancer using conventional ultrasound and sonoelastography.

\begin{tabular}{lcccc}
\hline Suspicious area, B-mode & $113 / 366$ & & & \\
Suspicious area, SE & $157 / 366$ & & & \\
Tumour foci, histology & $232 / 366$ & & & \\
& Sensitivity & Specificity & PPV & NPV \\
& $33 \%(27-40)$ & $74 \%(65-81)$ & $70 \%(61-78)$ & $38 \%(32-44)$ \\
B-mode $(95 \% \mathrm{CI})$ & $52 \%(45-58)$ & $73 \%(65-81)$ & $78 \%(70-84)$ & $46 \%(39-53)$ \\
$\mathrm{SE}(95 \% \mathrm{CI})$ & $59 \%(50-56)$ & $71 \%(61-80)$ & & \\
Size $\leq 40 \mathrm{cc}, \mathrm{n}=38$ & $42 \%(32-52)$ & $73 \%(65-81)$ & & \\
Size $>40 \mathrm{cc}, \mathrm{n}=23$ & $42 / 61(62 \%)$ & & & \\
Detection index tumour, b-mode & $56 / 61(92 \%)$ & & & \\
Detection index tumour, SE & $\mathrm{P}<0.005$ & & & \\
Chi-square-test & & & & \\
\hline
\end{tabular}

$\mathrm{SE}=$ sonoelastography, $\mathrm{CI}=$ confidence interval, $\mathrm{PPV}=$ positive predictive value, $\mathrm{NPV}=$ negative predictive value

a more precise detection of tumour infiltration with a sensitivity of $52 \%$ and a specificity of $74 \%$. Positiveand negative predictive values are shown in table II. In comparison to small prostate volumes, the sensitivity for glands larger than $40 \mathrm{~cm}^{3}$ was reduced.

Due to the low number of patients with extracapsular prostate cancer growth in our collective no clear conclusion can be drawn for the worthiness of SE in terms of predicting extracapsular extension or seminal vesicle infiltration.

\section{Discussion}

In accordance to previous single centre reports, our data underline the potential capacity of prostate SE for prostate cancer assessment. A closer view at the currently available clinical reports on SE shows that the results are either based on the comparison of preoperative prostate imaging and postoperative histological cancer localisation or on clinical trials with patients undergoing elastography-guided biopsies according to specific pro- 
Medical Ultrasonography 2012; 14(3): 182-186

\begin{tabular}{|c|c|c|c|c|c|}
\hline Author & Journal & $\mathbf{n}$ & Design & Sensitivity /specificity & Device \\
\hline Salomon et al. & Eur Urol (2008) & 109 & comparison SE/ histology & $75.4 \% / 76.6 \%$ & Hitachi \\
\hline Eggert et al. & Urologe (2008) & 351 & biopsy & $44,5 \% / 89 \%$ & Voluson, GE medical \\
\hline Pallwein et al. & Eur J Radiol (2008) & 492 & biopsy & $87 \% / 72 \%$ & Hitachi \\
\hline Tsutsumi et al. & $\begin{array}{c}\text { Int J Clin Oncol } \\
\text { (2007) }\end{array}$ & 51 & comparison SE/ histology & Sensitivity $57-94 \%$ & Hitachi \\
\hline Sumura et al. & Int J Urol (2007) & 17 & comparison SE/ histology & Sensitivity $74,1 \%$ & Hitachi \\
\hline
\end{tabular}

$\mathrm{n}=$ pacients' number

tocols. [4-9]. Relating to the used ultrasound devices, it is conspicuous that the majority of studies are based on Hitachi systems; only few available data rest upon other ultrasound systems and techniques. Table III illustrates the heterogeneity of published data regarding the sensitivity of SE.

With regard to technical variability, different systems of imaging and scaling represent a relevant difference. To obtain reproducible images during Siemens-SE the system implements a quality factor indicating the measure of (passive) pressure and examination quality. In our study, we observed a sensitivity of $53 \%$ and a positive predictive value of $78 \%$ for Siemens-SE. Especially the low sensitivity represents an unsatisfying result in relation to the requirements for local prostate cancer imaging and in comparison to previous studies and varying ultrasound devices.

In our opinion it is speculative whether these differences are based on a minor diagnostic power of the used SE device or potentially a lower examiner experience.

Previous publications agree with the statement that $\mathrm{SE}$ is an examiner dependant diagnostic tool $[4,9]$.

Despite a lack of available data regarding the practical use of the Siemens device, we were able to obtain a significant improvement of prostate cancer detection with SE compared to conventional transrectal ultrasound. Sedelaar et al. systematically reviewed the literature on prostate ultrasound revealing that the biopsy of a suspicious hypo-echogenic area detects prostate cancer in only $17-57 \%$ [10]. The results are in line with our own observations. The sensitivity for gray-scale ultrasound was low, accounting for only $33 \%$. As in the majority of conducted studies on prostate SE, cancer assessment was performed on an indirect basis, comparing preoperatively stored ultrasound pictures to postoperative pathological analysis. In order to increase the accuracy of analysing a suspicious electrographic region and to compare the practicability of SE-guided biopsies in contrast to diagnostic standard 1012 core biopsies, further clinical trials should follow.
Artefacts during SE are a common finding and represent a problem concerning the interpretation of examination results. In accordance to our own experience with Siemens-SE, sensitivity of elastography decreases with increasing prostate size and the presence of benign prostate hyperplasia (BPH) [5]. In our series, the observed sensitivity of $42 \%$ for glands larger than $40 \mathrm{~cm}^{3}$ was noticeably low in contrast to $59 \%$ sensitivity for prostates smaller than $40 \mathrm{~cm}^{3}$. Some reports quote that routinely appearing examination artefacts, like the "soft-rim-artefact", a soft layer surrounding the prostate tissue as an indication of the adventitial connective tissue, could predict capsular infiltration or extracapsular growth $[9,11]$. Since our own observations and the majority of available studies are based on the examination results of patients with merely organ confined disease, larger series are required to further validate these observations. The impact of prostate carcinoma differentiation on SE results is controversial. As Miyagawa and co-workers report from equality of diagnostic sensitivities of SE in relation to the Gleason score, a publication by Sumura et al. and the analysis of Salomon et al. showed an increased sensitivity for higher graded carcinoma $[5,9,12]$. In contrast, another Japanese single centre trial obtained best detection rates for low grade prostate cancer [8].

As most modern ultrasound devices offer a variety of examination techniques, combinations of e.g. SE, conventional gray-scale ultrasound, colour-Doppler or contrast enhanced ultrasound are generally feasible in order to raise detection rates. Myagawa et al. observed an improved detection of prostate cancer combining SE and conventional gray-scale transrectal ultrasound [5].

Magnetic resonance imaging (MRI) has been described as a valid instrument referring to local prostate cancer staging and MRI guided biopsy has demonstrated its potential value in clinical trials. Turkbey et al observed a sensitivity of $60.7 \%$ and a specificity of $72.7 \%$ for the prediction of prostate cancer applying multi-parametric MRI [13]. Recently, an expert panel established stand- 
ardized conduct and interpretation for the use of MRI in the diagnosis and staging of prostate cancer. Nevertheless, a general recommendation for the routine use of multi-parametric MRI should follow further validation in prospective studies [14-15].

With regard to costs, eligibility and the duration of the examination, SE is a promising alternative diagnostic tool with good feasibility in an ambulatory setting, which warrants further efforts in technical development and clinical investigation.

The investigator dependency of SE and the indirect approach of comparing preoperatively stored images to postoperative histological slides represent major limitations of our study.

\section{Conclusions}

Transrectal sonoelastography allows a more precise prostate cancer localisation than conventional ultrasound. SE may close diagnostic gaps in local staging and in the setting of prostate biopsy, especially in absence of extensive BPH. As the method is closely related to the experience of the examiner and the specifics of the used device and technique, studies are needed to evaluate these influences. Furthermore, it is worthwhile to determine the potential of SE in optimizing cancer detection during prostate biopsy.

\section{Acknowledgements: none.}

Conflicts of interest: none to declare.

\section{References}

1. National Comprehensive Cancer Network (NCCN). Prostate Cancer Early Detection. Clinical Practice Guidelines in Oncology. V.2.2007. Fort Washington: NCCN; 2007.

2. Eichler K, Hempel S, Wilby J, Myers L, Bachmann LM, Kleijnen J. Diagnostic value of systematic biopsy methods in the investigation of prostate cancer: a systematic review. J Urol 2006; 175: 1605-1612.

3. NHS Cancer Screening Programmes. Undertaking a transrectal ultrasound guided biopsy of the prostate. PCRMP
Guide No 1. Sheffield: NHS Cancer Screening Programmes; 2006.

4. Eggert T, Khaled W, Wenske S, Ermert H, Noldus J. Impact of elastography in clinical diagnosis of prostate cancer. A comparison of cancer detection between B-mode sonography and elastography-guided 10-core biopsies. Urologe A 2008; 47: 1212-1217.

5. Miyagawa T, Tsutsumi M, Matsumura T, et al. Real-time elastography for the diagnosis of prostate cancer: evaluation of elastographic moving images. Jpn J Clin Oncol 2009; 39: 394-398.

6. Nelson ED, Slotoroff CB, Gomella LG, Halpern EJ. Targeted biopsy of the prostate: the impact of color Doppler imaging and elastography on prostate cancer detection and Gleason score. Urology 2007; 70: 1136-1140.

7. Pallwein L, Mitterberger M, Struve P, et al. Real-time elastography for detecting prostate cancer: preliminary experience. BJU Int 2007; 100: 42-46.

8. Tsutsumi M, Miyagawa T, Matsumura T, et al. The impact of real-time tissue elasticity imaging (elastography) on the detection of prostate cancer: clinicopathological analysis. Int J Clin Oncol 2007; 12: 250-255.

9. Salomon G, Köllerman J, Thederan I, et al. Evaluation of prostate cancer detection with ultrasound real-time elastography: a comparison with step section pathological analysis after radical prostatectomy. Eur Urol 2008; 54: 1354-1362.

10. Sedelaar JP, Vijverberg PL, De Reijke TM, et al. Transrectal ultrasound in the diagnosis of prostate cancer: state of the art and perspectives. Eur Urol 2001; 40: 275-284.

11. Salomon G, Graefen M, Heinzer H, et al. The value of realtime elastography in the diagnosis of prostate cancer. Urologe A 2009; 48: 628-636.

12. Sumura M, Shigeno K, Hyuga T, Yoneda T, Shiina H, Igawa $\mathrm{M}$. Initial evaluation of prostate cancer with real-time elastography based on step-section pathologic analysis after radical prostatectomy: a preliminary study. Int J Urol 2007; 14: 811-816.

13. Turkbey B, Xu S, Kruecker J, et al. Documenting the location of systematic transrectal ultrasound-guided prostate biopsies: correlation with multi-parametric MRI. Cancer Imaging 2011; 11: 31-36.

14. Dickinson L, Ahmed HU, Allen C, et al. Magnetic resonance imaging for the detection, localisation, and characterisation of prostate cancer: recommendations from a European consensus meeting. Eur Urol 2011; 59: 477-494.

15. Heidenreich A. Consensus criteria for the use of magnetic resonance imaging in the diagnosis and staging of prostate cancer: not ready for routine use. Eur Urol 2011; 59: 495-497. 